\title{
Correspondence
}

\section{Routine enquiry for domestic violence is not enough}

We read with great interest the paper by Morgan et al on the prevalence of domestic violence and acceptability of clinical enquiry about abuse among female psychiatric patients. This study is highly topical, as our own review of the academic literature identified a dearth of research on prevalence of domestic violence in psychiatric settings and low rates of staff detection in routine clinical practice, particularly in the UK. ${ }^{2}$ Morgan et al's paper reported that the majority of patients sampled perceived clinical enquiry about domestic violence as acceptable. This finding, alongside high reported prevalence rates, led the authors to advocate routine enquiry about domestic violence by mental health professionals.

However, although our review found that the introduction of routine clinical enquiry in mental health services is associated with an increase in clinician identification of domestic violence, we do not believe that sufficient evidence currently exists to justify its implementation, unless it is introduced with training on how to ask, and is carried out with a referral and care pathway that can address the domestic violence. As well as Morgan et al, we have highlighted that, to date, research on the effectiveness of screening for domestic violence has not found evidence that enquiry leads to reductions in patient morbidity. Furthermore, routine enquiry is not a benign intervention and can lead to adverse consequences. ${ }^{3}$ The report from the Department of Health Violence Against Women and Children (VAWC) National Health Service (NHS) taskforce ${ }^{4}$ has also stressed the importance of prior clinical training and care pathways for domestic violence in ensuring efficacy of routine clinical enquiry. The Department of Health delivered an NHS awareness-raising campaign to coincide with End Violence against Women Day on 25 November 2010. This has led to support for primary care trusts and NHS trusts to raise the profile of VAWC locally. We hope that all mental health trusts will take advantage of the associated resources that have been sent to all trusts to raise awareness among staff and their local communities to address this highly prevalent issue for our patients.

Declaration of interest The authors receive funding for a project on domestic violence and mental health from the NIHR Research for Patient Benefit programme. L.H. is a member of the Department of Health Implementation Group on Violence Against Women and Children. Neither of these organisations was involved with this letter at any stage.

1 Morgan JF, Zolese G, McNulty J, Gebhardt S. Domestic violence among female psychiatric patients: cross-sectional survey. Psychiatrist 2010; 34: $461-4$

2 Howard LM, Trevillion K, Khalifeh H, Woodall A, Agnew-Davies R, Feder $\mathrm{G}$. Domestic violence and severe psychiatric disorders: prevalence and interventions. Psychol Med 2010; 40: 881-93.
3 Bacchus LJ, Aston G, Murray SF, Virolas CT, Jordan P. Evaluation of an Innovative Multi-Agency Domestic Violence Service at Guy's and St. Thomas' NHS Foundation Trust. King's College London (www.kcl.ac.uk/content/1/c6/05/72/39/ Evaluationsummaryleaflet.pdf).

4 Taskforce on the Health Aspects of Violence Against Women and Children. Responding to Violence Against Women and Children - The Role of the NHS. The Report from the Taskforce on the Health Aspects of Violence Against Women and Children. Department of Health, 2010.

Louise M. Howard is Professor of Women's Mental Health and consultant psychiatrist, Section of Women's Mental Health, PO31, Institute of Psychiatry, King's College London, and South London and Maudsley NHS Foundation Trust, email: louise.howard@kcl.ac.uk; Kylee Trevillion is researcher, Section of Women's Mental Health, King's College London

doi: 10.1192/pb.35.2.74

\section{The heart of psychiatry}

Craddock et al's ${ }^{1}$ attempt to define the core expertise of psychiatry is timely and welcome, but disconcertingly incomplete. Ethics, history and philosophy are no less central to the psychiatrist's craft. We have, after all, chosen to care for the only organ in the body that can vote.

Ethical issues arise all over medicine, but in psychiatry they abound. Issues of agency, belief and capacity, daily juggling the paradox of coercion and compassion, define much of our practice. No other branch of medicine has an entire legal statute devoted to it.

History is just as crucial. Ideas of illness, suffering and disease change constantly with the values and wisdom of the times and awareness of the progress of ideas over time is essential to the refinement of our practice. Medicine privileges us with a chance to study this within a living system of art and science, 3000 years in the making.

The importance of these skills is evident in doctors' relationship with society. Upon qualification, we receive honorary doctorate for nothing more than a bachelor degree (not unlike an increasing number of psychological therapists). Our title acknowledges that we have chosen to go where others fear to tread; severe mental illness is one of the most perplexing matters of all.

An omission of these issues from any definition of our craft may explain the difficulties that psychiatry apparently faces today. ${ }^{2}$ Much of the concern about mental illness over the past two decades has centred on the ethics of coercion in risky cases and transgressions of the indefinable border between illness and 'healthy' distress. Psychiatry, practised properly, with its unique ability to evaluate past and present; brain, mind and body; culture, danger and bus pass ${ }^{3}$ brings a clarity to these debates that none can rival.

This view appears anathema in a culture that places such heavy emphasis on consensus and certainty. The measures, goals and guidelines that abound in modern practice are symptoms of this. Against such apparent certainty, more subtle - and far more important - values become ever harder to define, but we omit them from our accounts at our peril. 